\title{
Holmes Tremor After Brainstem Hemorrhage, Treated With Levodopa
}

\author{
Jae Hyun Woo, MD, Bo Young Hong, MD, Joon Sung Kim, MD, Seok Ho Moon, MD, \\ Soo Yeon Kim, MD, Hye Young Han, MD, Dong Yoon Park, MD, Seong Hoon Lim, MD
}

Department of Rehabilitation Medicine, St. Vincent Hospital, The Catholic University of Korea College of Medicine, Suwon, Korea

Holmes tremor is a rare movement phenomenon, with atypical low-frequency tremor at rest and when changing postures, often related to brainstem pathology. We report a 70-year-old female patient who was presented with dystonic head and upper limb tremor after brainstem hemorrhage. The patient had experienced a sudden onset of left hemiparesis and right facial paralysis. Brain magnetic resonance imaging showed an acute hemorrhage from the brachium pontis through the dorsal midbrain on the right side. Several months later, the patient developed resting tremor of the head and left arm, which was exacerbated by a sitting posture and intentional movement. The tremor showed a regular low-frequency $(1-2 \mathrm{~Hz})$ for the bilateral sternocleidomastoid and cervical paraspinal muscles at rest. The patient's symptoms did not respond to propranolol or clonazepam, but gradually improved with levodopa administration. Although various remedies were attempted, overall, the results were poor. We suggest that levodopa might be a useful remedy for Holmes tremor. The curative or relieving effect of the dopaminergic agent in Holmes tremor needs more research.

Keywords Holmes tremor, Traumatic brain stem hemorrhage, Levodopa

\section{INTRODUCTION}

In 1904, Gordon Holmes described a unique motor abnormality, characterized by low-frequency tremor at rest, which was exacerbated by specific postures [1]. Holmes tremor (HT) is a rare, symptomatic tremor of lowfrequency; mostly below $4.5 \mathrm{~Hz}$. It typically involves the

Received July 25, 2012; Accepted October 17, 2012

Corresponding author: Seong Hoon Lim

Department of Rehabilitation Medicine, St. Vincent Hospital, The Catholic University of Korea College of Medicine, 93 Jungbu-daero, Paldal-gu, Suwon 442-723, Korea

Tel: +82-31-249-7650, Fax:+82-31-251-4481, E-mail: seonghoon@ catholic.ac.kr

(c) This is an open-access article distributed under the terms of the Creative Commons Attribution Non-Commercial License (http://creativecommons. org/licenses/by-nc/3.0) which permits unrestricted noncommercial use, distribution, and reproduction in any medium, provided the original work is properly cited.

Copyright (C) 2013 by Korean Academy of Rehabilitation Medicine proximal limbs and arises as a delayed manifestation of lesions in the upper brainstem, often related to stroke or trauma. The Consensus Statement of the Movement Disorder Society on tremor, published in 1998, described that both the dopaminergic nigrostriatal and the cerebello-thalamic systems must be involved for the occurrence of this type of tremor [2]. Recently, HT associated with a brainstem lesion has been reported as an extremely rare tremor, along with drug-induced tremor, orthostatic tremor, and psychogenic tremor [1]. In Korea, a case with HT and palatal tremor after bilateral brainstem hemorrhage was reported [3]. However, the case report was not sufficient to determine appropriate treatment for HT because of the patient's poor medical condition. To date, there is no reported controlled interventional study. Pharmacological treatment is usually disappointing, and surgical procedures, such as stereotactic thalamotomy 
or thalamic stimulation, are often required for refractory cases [4-6].

In the present case, the patient who was diagnosed with HT after a brainstem hemorrhage, showed significant improvement after treatment with a dopaminergic agent.

\section{CASE REPORT}

A 70-year-old woman who had hypertension developed left side hemiparesis, right facial paralysis, severe dysarthria, and left hemiataxia. Physical examination revealed dysmetria and mildly increased deep tendon reflexes in the left upper and lower limbs. She did not show rigidity. Sensory examination demonstrated left hypesthesia. Brain magnetic resonance imaging showed an acute

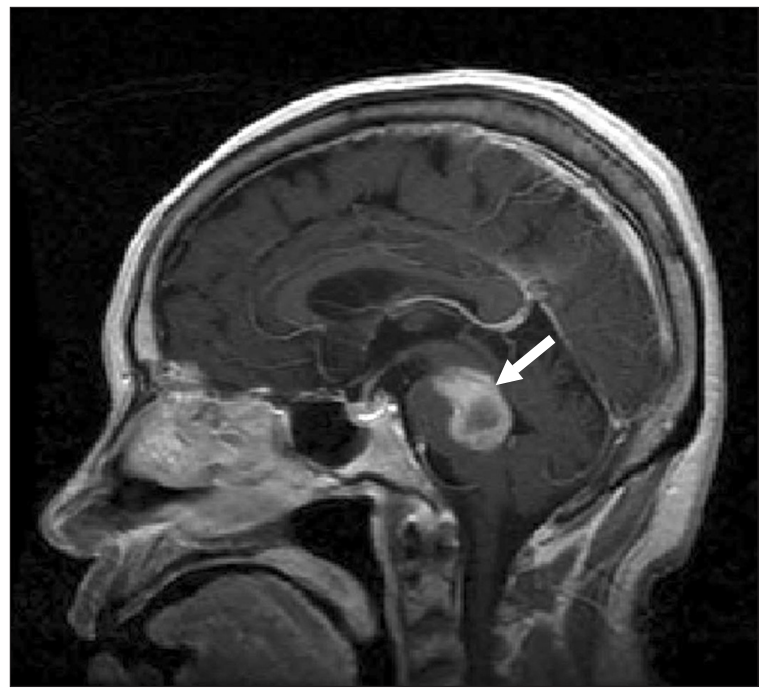

Fig. 1. Acute hemorrhage (arrow) was seen on sagittal brain $\mathrm{T} 1$ magnetic resonance imaging scan, extending from the brachium pontis to the dorsal midbrain. hemorrhage from the brachium pontis through the dorsal midbrain on the right side (Fig. 1).

Prior to the tremor, her Modified Barthel Index (MBI), which was used to assess her activities of daily living, was 45 due to the left hemiparesis. At 6 months from the initial hemorrhage, the patient developed resting tremor of the head and left arm, which worsened progressively in the following days. As her motor function was impaired, it was difficult for her to sit up and hold a spoon, and this hindered eating independently. Her cognitive level was 26 , which is considered within normal range based on the Mini Mental State Examination.

The patient's MBI score decreased to 30 . Tremor continued with a sitting posture, particularly when her arms approached an object, and it was worsened by emotional distress, and ceased during sleep. The patient also developed a torticollis to the right and a regular dystonic head tremor, which was characterized by left chin rotation and right lateral head tilt. We measured the frequency of tremor using electromyography (EMG) equipment (VikingSelect; Nicolet, Madison, WI, USA). Recordings were made with a pair of surface EMG electrodes. The active (recording) electrode was placed over the bilateral sternocleidomastoid or the $\mathrm{C} 7$ paraspinal muscles. The reference electrode was positioned on the mastoid process of the temporal bone. The tremor of the head showed a slow (1.1 Hz), regular frequency at rest (Fig. 2). We prescribed propranolol (Indenol; DongKwang Pharm, Seoul, Korea), the first-line drug for essential tremor, at a dose of $120 \mathrm{mg}$ per day and increased this to $240 \mathrm{mg}$ per day for 2 weeks. However, her symptoms persisted. Next, she was recommended with other medications, namely, clonazepam and levetiracetam. She took several doses of clonazepam (Rivotril; Roche, Basel, Switzerland) from 0.5 to $1 \mathrm{mg}$;

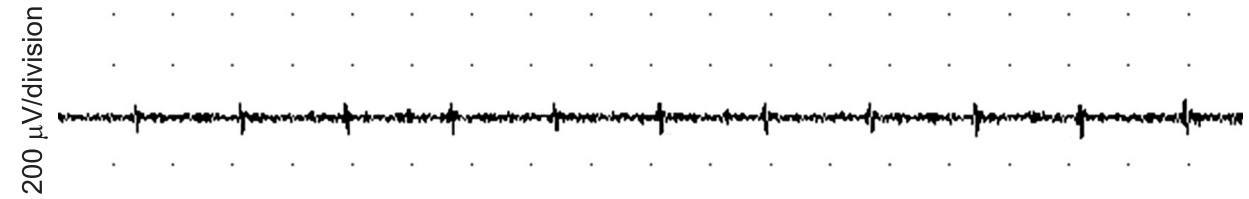

500 ms/division
Fig. 2. Initial electromyogram of the right sternocleidomastoid muscle of the neck showed regular, low-frequency $(1.1 \mathrm{~Hz})$ contraction $(200 \mu \mathrm{V}$ per division and $500 \mathrm{~ms}$ per division). 
however, the tremor was unchanged. After administration of levetiracetam (Keppra; UCB, Slough, UK), from 500 to $1,000 \mathrm{mg}$ per day for 2 weeks, her tremor showed some improvement. However, she complained of severe headache and mood changes; thus, she stopped taking levetiracetam. Next, she was treated with a levodopacontaining agent Stalevo (Novartis Korea, Seoul, Korea), starting at a dose of $200 \mathrm{mg}$ once a day and progressively increasing over a period of 1 month to reach a final dose of $200 \mathrm{mg}$, three times a day. One week after beginning the medication, her tremor lessened gradually. At the final dose of the drug, her tremor was diminished and she was discharged from the hospital. We also observed an improvement in her motor function; she was able to sit up independently. In addition, her ability to perform activities of daily living improved; the functional levels for eating and dressing were enhanced, with her MBI score improving to 46 .

A month later, the patient returned to our outpatient clinic and the tremor was significantly improved. Six months later, the effect continued and the tremor was considered successfully controlled.

\section{DISCUSSION}

HT is a resting, intentional, and sometimes postural tremor with a frequency of $4.5 \mathrm{~Hz}$ and below $[1,3]$. It is usually arrhythmic and has a variable delay, from 4 weeks to 2 years between the lesion and the first occurrence of tremor. The features of the tremor in our patient were consistent with the reported characteristics of HT [2]: 1) predominantly unilateral tremor; 2) present not only at rest but also when sitting and during movement, but disappearing during sleep; 3) low-frequency at $1.1 \mathrm{~Hz}$; and 4) occurring 6 months after a brainstem hemorrhage.

Two distinct neural pathways have been proposed as pathophysiological bases for HT. One involves the substantia nigra or nigrostriatal dopaminergic fibers, causing dopaminergic denervation of the striatum [7]. Remy et al. [8] found a marked decrease in 18F-dopa re-uptake in the caudate and putamen in a patient with HT due to a midbrain lesion, likely reflecting the ipsilateral severe striatal dopaminergic denervation. Such dopaminergic denervation may play a role in resting tremor, like that in Parkinson disease [7]. The structure involved in the genesis of HT may be the dentate projection pathway, one of non-dopaminergic structures. The ascending arm of this pathway includes the dentate-rubral and dentate-thalamic tracts; the descending arm includes the dentateolivary and dentate-rubro-olivary tracts, which form the rubro-olivo-cerebellar-rubral circuits. Interruption of these circuits may be responsible for postural and intentional tremors [1]. Midbrain lesions, such as hemorrhage, ischemia, and neoplasm may interrupt elements of both dopaminergic and non-dopaminergic pathways, producing the characteristic combination of resting, postural, and kinetic tremors seen in HT. This complex mechanism may account for the response variability to pharmacotherapy. Isolated cases of partial or complete relief have been reported with several drugs, including propranolol, clonazepam, and levetiracetam [6]. However, medical treatment is typically unsatisfactory, and both deep brain stimulation and ablation of the thalamus structures are often considered to provide the best results [5]. Many treatment methods have been tried; however, there is no gold-standard treatment for HT.

Some isolated cases of treatment with levodopa have occasionally been reported $[9,10]$. However, the therapeutic response to levodopa is variable. In addition, several studies have reported that patients with typical resting tremor do not respond to levodopa therapy; whereas, other patients with predominantly postural and intentional tremor responded satisfactorily. These clinical aspects indicate that a complex pathological mechanism may exist in HT, and furthermore, dopaminergic dysfunction is a prerequisite for the persistence of postural and intentional tremors. The finding that levodopa improves HT supports the suggestion that the dopaminergic pathway is involved (the substantia nigra, nigrostriatal fibers, or both) $[7,8]$. The postural component of HT could be related to disruption of the cerebellar outflow pathway (dentate-rubral and dentate-thalamic tracts) at the superior cerebellar peduncle, and the rubro-olivary tract in the central tegmentum [10]. In our case, the patient experienced a hemorrhage in the dorsal midbrain. Due to the location of the lesion, it was considered likely that both dopaminergic and cerebellar outflow pathways were impaired. Consequently, our patient showed marked improvements in resting, postural, and action tremors.

In conclusion, levodopa is an easy-to-test drug that may improve the symptoms in patients with HT. Remarkable results of levodopa in this patient demonstrate that 
dopaminergic denervation should not be underestimated in such HT cases, and dopaminergic agents should be tried in patients with HT before considering invasive therapies, such as deep brain stimulation. However, further studies are necessary to clarify its long-term efficacy and mechanism of action.

\section{CONFLICT OF INTEREST}

No potential conflict of interest relevant to this article was reported.

\section{ACKNOWLEDGEMENT}

The authors wish to acknowledge the financial support of the Catholic Medical Center Research Foundation made in the program year of 2012.

\section{REFERENCES}

1. Deuschl G, Bain P, Brin M. Consensus statement of the Movement Disorder Society on Tremor. Ad Hoc Scientific Committee. Mov Disord 1998;13 Suppl 3:223.

2. Deuschl G, Bergman H. Pathophysiology of nonparkinsonian tremors. Mov Disord 2002;17 Suppl 3:S41-8.

3. Lee GH, Kim SJ, Yoon JS, Jo JM. Bilateral hypertrophic olivary degeneration with oculopalatal tremor after brainstem hemorrhage: a case report. J Korean Acad Rehabil Med 2010;34:96-8.

4. Zeuner KE, Deuschl G. An update on tremors. Curr Opin Neurol 2012;25:475-82.

5. Nikkhah G, Prokop T, Hellwig B, Lucking CH, Ostertag CB. Deep brain stimulation of the nucleus ventralis intermedius for Holmes (rubral) tremor and associated dystonia caused by upper brainstem lesions: report of two cases. J Neurosurg 2004;100:1079-83.

6. Striano P, Elefante A, Coppola A, Tortora F, Zara F, Minetti C, et al. Dramatic response to levetiracetam in post-ischaemic Holmes' tremor. J Neurol Neurosurg Psychiatry 2007;78:438-9.

7. Defer GL, Remy P, Malapert D, Ricolfi F, Samson Y, Degos JD. Rest tremor and extrapyramidal symptoms after midbrain haemorrhage: clinical and 18Fdopa PET evaluation. J Neurol Neurosurg Psychiatry 1994;57:987-9.

8. Remy P, de Recondo A, Defer G, Loc'h C, Amarenco $\mathrm{P}$, Plante-Bordeneuve V, et al. Peduncular 'rubral' tremor and dopaminergic denervation: a PET study. Neurology 1995;45(3 Pt 1):472-7.

9. Raina GB, Velez M, Pardal MF, Micheli F. Holmes tremor secondary to brainstem hemorrhage responsive to levodopa: report of 2 cases. Clin Neuropharmacol 2007;30:95-100.

10. Velez M, Cosentino C, Torres L. Levodopa-responsive rubral (Holmes') tremor. Mov Disord 2002;17:741-2. 